\title{
Development of a New Plasma Diagnostic of the Critical Surface and Studies of the Ion Acoustic Decay Instability Using Collective Thomson Scattering
}

Final Report for Contract DOE-FG03-91SF18861

$$
\text { by }
$$

K. Mizuno and J. S. DeGroot, University of California, Davis

W. Seka, Laburatory of Laser Energetics, and

R. P. Drake, Lawrence Livermore National Laboratory

\section{MASTFR}




\title{
Table of Content
}

I. Summaries 3

II. Developments of the collective Thomson scattering

diagnostics for the ion acoustic decay instabilities

III. Experimental Arrangements and the CTS experiments

IV. Studies of the Ionic Charge State of High Z plasma

V. The Ion Acoustic Decay Instability in

High Power Laser Regime

VI. Studies of laser absorption for mederate

to high power irradiations

VII. References

VIII. Figure Captions

\section{DISCLAIMER}

\begin{abstract}
This report was prepared as an account of work sponsored by an agency of the United States Government. Neither the United States Government nor any agency thereof, ior any of their employees, makes any warranty, express or implied, or assumes any legal liability or responsibility for the accuracy, completeness, or usefulness of any information, apparatus, product, or process disclosed, or represents that its use would not infringe privately owned rights. Reference herein to any specific commercial product, process, or service by trade name, trademark, manufacturer, or otherwise does not necessarily constitute or imply its endorsement, reconmendation, or favoring by the United States Government or any agency thereof. The views and opinions of authors expressed herein do not necessarily state or reflect those of the United States Government or any agency thereof.
\end{abstract}




\section{Summaries}

We have developed 5-channel collective Thomson scattering system to measure the ion acoustic wave excited by the ion acoustic wave decay instabilities. The multichannel collective Thomson scattering technique was established with $4 \omega$ probe laser beam using GDL laser system at LLE, Univ. of Rochester.

We have obtained the ionic charge state $\mathrm{Z}$ by measuring the second harmonic emission from the ion acoustic decay instability. The LASNEX computer simulation calculations have been carried out. The experimental results agree very well with the LASNEX computer simulation results with the flux number $\mathrm{f}=\mathrm{C} .1$.

In high power laser regime, the spectrum become broad, and the $\Delta \lambda$ decreases indicating that the turbulent like spectrum is observed.

In order to understand the experimental results, we have developed a theory to study absorption of laser and heat transport. This new theory includes the temporal evolution of the heat conduction region. The results agree with flux-limited hydrodynamic simulations. 


\section{Developments of the collective Thomson scattering diagnostics for the ion acoustic decay instabilities.}

\section{Measurement of Electron Temperature using Thomson Scattering -. Theoretical Considerations}

Collective Thomson scattering is a three wave process satisfying the phase-matching

conditions $\overrightarrow{\mathrm{k}}_{\mathrm{s}}=\overrightarrow{\mathrm{k}}_{\mathrm{O}} \pm \overrightarrow{\mathrm{k}}_{\mathrm{p}}$ (see Fig. 2 ) and the energy conservation $\omega_{\mathrm{s}}=\omega_{\mathrm{O}} \pm \Omega_{\mathrm{i}}$. The $k$ 's and $\omega$ 's are the wave vectors and frequencies of the three waves; the subscripts o and $s$ refer to the incident and scattered electromagnetic waves for a probe beam, and the subscript $p$ refers to a longitudinal plasma wave which, in our case, is an ion-acoustic wave generated by the IADI instability. Since $\Omega_{\mathrm{i}} \ll \omega_{\mathrm{S}}$ or $\omega_{\mathrm{O}}$, we have $\left|\mathrm{k}_{\mathrm{S}}\right| \approx\left|\mathrm{k}_{\mathrm{O}}\right|$ and

$$
\mathrm{k}_{\mathrm{p}} \approx 2 \mathrm{k}_{\mathrm{O}} \sin (\theta / 2)
$$

where $\theta$ is the scattering angle $\alpha=\pi-\theta$, and $\beta=\alpha / 2$. The frequency of the ion-acoustic wave is determined by the dispersion relation

$$
\begin{aligned}
& \Omega_{i}=k_{p} c_{s}=k_{p} \sqrt{\left(T_{e} / M\right)\left(Z+3 T_{i} / T_{e}\right)}, \text { for } k_{p} \lambda \text { De } \ll 1 \\
& \approx k_{p} \sqrt{Z T_{e} / M}, \text { for high } Z \text { targets, }
\end{aligned}
$$

where $Z_{i}$ is the average ionic charge, $T_{e}$ and $T_{i}$ are the thermal electron and ion temperatures, and $\mathrm{M}$ is the average ion mass. For low $\mathrm{Z}$ targets such as $\mathrm{CH}, \Omega_{\mathrm{l}}$ depends only very weakly on the electron-ion temperature ratio, while for high $\mathrm{Z}$ targets this effect is negligible.

A good measure of the thermal electron temperature can thus be obtained from a simultaneous measurement of $\Omega_{\imath}$ and $\theta$, i.e. from a simultaneous measurement of the spectrum and the scattering angle. This forms the basis for our proposed electron temperature measurements. 
To aid the design of the experiments, we can estimate the appropriate scattering angles for the electron temperatures expected in our experiments by using Eq. (1) in conjunction with our past observations that the most prevalent values for $k_{i}$ are found near the Landau damping cut-off, $\mathrm{k}_{\mathrm{i}} \lambda \mathrm{D} \approx 0.25$. This yields the simple relationship

$$
\sin (\theta / 2)=\sqrt{T_{\min } / T_{e}}
$$

where $T_{\min } \approx 500 \mathrm{eV}$ and $890 \mathrm{eV}$ for a prote beam of frequency $\omega_{\mathrm{O}}=4 \omega_{\mathrm{L}}$ and $3(\mathrm{LL}$, with $\omega_{\mathrm{L}}$ being the frequency of the plasma-producing laser. This relationship (Eq. (3)) is also plotted in Fig. 1 which clearly shows that good electron temperature measurements can be expected for large angle scattering of the probe beam.

Note that Eq. (3) is used solely for the purpose of estimating the appropriate range of scattering arigles for the proposed experiments, while the actual determination of the electron temperature is made by simultaneously measuring $\omega_{i}$ and $\theta$ and using Eq. (2) as outlined above.

\section{a. Scattered Power}

The required energy of the probe beam is estimated using Thomson scattering theory. For the averaged scattered power in the frequency range $\omega_{S} \rightarrow \omega_{S}+d \omega_{S}$ and within the solid angle $d \Omega$ centered on $\vec{R}$, we have

$$
\delta P_{S}\left(\vec{R}, \omega_{S}\right) d \Omega d \omega_{S}=\frac{P_{O} r^{2} O}{2 \pi} d \Omega d \omega_{S}\left|\vec{s} \times \vec{s} \times \frac{\vec{E} i 0}{\left|E_{i o}\right|}\right|^{2} \cdot n_{e o} L \cdot S(\vec{k}, \omega)
$$

and the spectral density function is defined as

$$
S(\vec{k}, \omega) \equiv \lim _{T \rightarrow \infty} \frac{1}{\left|n_{e}(\vec{k}, \omega)\right|^{2}} \frac{\mid n_{\text {eo }}}{n^{2}}
$$

where $P_{0}$ is the incident power of the probe beam, $r_{0}^{2}=e^{4 / m_{O}^{2}} c^{4}$ is the Thomson scattering cross section, $\vec{s}$ is the unit vector in the direction of the scattering, and $L$ is the length of the interaction region. The time and space averaged ion acoustic wave amplitude may be written using the spectral density function, 


$$
\frac{\left|\mathrm{n}_{\mathrm{e}}(\overrightarrow{\mathrm{r}}, \mathrm{t})\right|^{2}}{\mathrm{n}_{\mathrm{e} o}}=\left(\frac{1}{2 \pi}\right)^{4} \frac{1}{\mathrm{n}_{\mathrm{eo}}} \int_{-\infty}^{\infty} \mathrm{d} \omega \int_{-\infty}^{\infty} \mathrm{d} \overrightarrow{\mathrm{k}} \mathrm{S}(\overrightarrow{\mathrm{k}}, \omega)
$$

Assuming that $S \overrightarrow{\mathrm{k}}$ is constant within a frequency range around $\Omega_{\mathrm{i}}$ and the wavelength spread is $\Delta \mathrm{k}_{\mathrm{x}}, \Delta \mathrm{k}_{\mathrm{y}}, \Delta \mathrm{k}_{\mathrm{z}}$, and zero outside, we obtain the scattered power in the solid angle $\Delta \Omega$ from Eqs. (4) and (6)

$$
\mathrm{P}_{\mathrm{S}}=\left.(2 \pi)^{3} \mathrm{P}_{\mathrm{O}} \mathrm{r}_{\mathrm{o}} \Delta \Omega \mathrm{n}_{\mathrm{eO}}^{2}\left|\overrightarrow{\mathrm{s}} \times\left(\overrightarrow{\mathrm{s}} \times \frac{\overrightarrow{\mathrm{E}}}{\left|\mathrm{E}_{\mathrm{iO}}\right|}\right)^{2} \cdot \mathrm{L} \cdot \frac{1}{\Delta \mathrm{k}_{\mathrm{x}} \Delta \mathrm{k}_{\mathrm{y}} \Delta \mathrm{k}_{\mathrm{z}}} \cdot\right| \frac{\mathrm{n}_{\mathrm{e}}}{\mathrm{n}_{\mathrm{eO}}}\right|^{2}
$$

In order to obtain an order of magnitude estimate, we assume approximate values $\Delta \mathrm{k}_{\mathrm{x}, \mathrm{y}, \mathrm{z}}$ $\sim 2 \pi / \lambda_{0}$ ( $\lambda_{0}$ is the wavelength of the probe beam). The wave number of the ion acoustic wave $k_{p}$ is of order $k_{0}$, because of the large scattering angle in our experiments. We obtain the following approximate value of the normalized scattered power

$$
\mathrm{P}_{\mathrm{S}} / \mathrm{P}_{\mathrm{O}} \approx \mathrm{fr}^{2}{ }_{\mathrm{o}} \mathrm{n}^{2} \mathrm{eo} \Delta \Omega \cdot \mathrm{L} \cdot \lambda^{3} \mathrm{O}\left|\frac{\mathrm{n}_{\mathrm{e}}}{\mathrm{n}_{\mathrm{eo}}}\right|^{2}
$$

where $f$ is the fraction of the incident power interacting with the ion acoustic waves. We obtain a scattered power of $\mathrm{P}_{\mathrm{S}} \approx 1 \times 10^{5}$ watt for our experimental parameters, $\left|\frac{\mathrm{n}_{\mathrm{e}}}{\mathrm{n}_{\mathrm{eo}}}\right|^{2}=$ $10^{-4}, \mathrm{~L}=100 \mu \mathrm{m}, \mathrm{f}=0.1, \lambda_{\mathrm{o}}=1 / 4 \mu \mathrm{m}, \mathrm{n}_{\mathrm{eo}}=10^{21} \mathrm{~cm}^{-3}, \Delta \Omega / 4 \pi=10^{-3}$, and $\mathrm{P}=10^{9} \mathrm{~W}$ (which corresponds to a $100 \mathrm{~mJ}$ and $100 \mathrm{psec}$ probe beam). The estimated number of scattered photon is about $10^{11}$ for the $100 \mathrm{psec}$ probe bearm. The Bremsstrahlung emission is given by

$$
\mathrm{P}_{\mathrm{B}}(\mathrm{W})=1.9 \times 10^{-28} \frac{\mathrm{n}_{\mathrm{e}} \mathrm{n}_{\mathrm{i}} \mathrm{Z}^{2}}{\lambda^{2}(\AA) \mathrm{T}_{\mathrm{e}}^{1 / 2}(\mathrm{eV})} \exp \left(\frac{-\mathrm{hc}}{\lambda \mathrm{T}_{\mathrm{e}}}\right) \cdot \mathrm{V}_{\mathrm{p}} \cdot \frac{\Delta \Omega}{4 \pi} \cdot \Delta \lambda(\stackrel{\AA}{\mathrm{A}})
$$

We obtain $\mathrm{PB}_{B}(W) \approx 2$ watt for $\mathrm{T}_{\mathrm{e}}=1 \mathrm{keV}$, a plasma volume $\mathrm{V}_{\mathrm{p}} \approx 6 \times 10^{-5} \mathrm{~cm}^{3}$ (the width $300 \mu \mathrm{m}$ and diameter $500 \mu \mathrm{m}), \Delta \lambda=10 \AA$, solid angle $\Delta \Omega / 4 \pi=10^{-3}$, and plasma

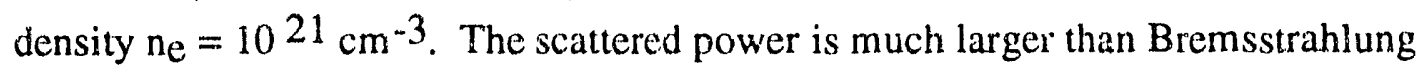


emission $\left(\mathrm{P}_{\mathrm{S}} / \mathrm{PB}_{\mathrm{B}}-10^{3}\right)$. We have also estimated the scattering power, $\mathrm{P}_{\mathrm{S}}$, th, from the thermal electrons, which is given by

$$
\mathrm{P}_{\mathrm{S}, \mathrm{th}}(\mathrm{W})=\sigma_{\mathrm{T}} \cdot \mathrm{I}_{\mathrm{O}} \cdot \mathrm{n}_{\mathrm{e}} \cdot \mathrm{V}_{\mathrm{L}} \cdot \frac{\Delta \Omega}{4 \pi} \cdot \mathrm{S}(0) \cdot \Delta \lambda
$$

and

$$
S(0)=\frac{45}{T^{1 / 2} e^{2} \lambda_{0} \sin \frac{\theta}{2}}
$$

We obtain $\mathrm{P}_{\mathrm{s}, \mathrm{th}} \approx 0.3 \mathrm{~W}$ for $\sigma \mathrm{Tot}=6.65 \times 10^{-25} \mathrm{~cm}^{2}, \mathrm{I}_{\mathrm{O}}=10^{12} \mathrm{~W} / \mathrm{cm}^{2}, \mathrm{n}_{\mathrm{e}}=10^{21} \mathrm{~cm}^{-3}$, $\mathrm{V}=6 \times 10^{-5} \mathrm{~cm}^{3}, \mathrm{~d} \Omega / 4 \pi=10^{-3}, \Delta \lambda=10\left(^{\circ}, \mathrm{A}\right), \mathrm{T}_{\mathrm{e}}=1 \mathrm{keV} . \mathrm{P}_{\mathrm{S}, \text { th }}$ is slightly smaller than Bremsstrahlung emission.

The photon number in the scattered probe beam $\left(\sim 10^{11}\right)$ is much larger than the minimum number required for detection $\left(10^{5} \sim 10^{6}\right)$, and the scattered power is also much larger than Bremsstrahlung emission $\left(\mathrm{P}_{\mathrm{S}} / \mathrm{P}_{\mathrm{B}}-10^{3}\right)$. Therefore, collective Thomson scattering from the ion acoustic waves can be measured in our experiments.

\section{b. Experimental Accuracy}

An estimate of the accuracy of the electron temperature measurement is obtained from Eq. (1):

$$
\frac{\Delta \mathrm{T}_{\mathrm{e}}}{\mathrm{T}_{\mathrm{e}}}=\frac{\cos \left(\pi-\frac{\alpha}{2}\right)}{\sin \left(\pi-\frac{\alpha}{2}\right)} \times \Delta \mathrm{c}
$$

The estimated uncertainty $\left|\Delta \mathrm{T}_{\mathrm{e}} / \mathrm{T}_{\mathrm{e}}\right|$ for our typical parameters is about $8 \%$ with a solid angle $\Delta \Omega / 4 \pi=10^{-3}$ and $\alpha=75^{\circ}$. We have designed our experiments to have a large scattering angle so that uncertainty in the measure electron temperature is minimized.

Ion acoustic waves are excited by the IADI. It is expected that the ion acoustic waves have a significant width in wave number and propagation angel. However, those widths will not induce any uncertainty in the electron temperature measurement, because we 
measure the scattered angle and the frequency of the scattered signal simultaneously (each scattering angle corresponds to one frequency).

The advantage of our technique is that we do not have to know the absolute value of the scattered power to obtain the electron temperature. The measurements do not depend on the intensity of the probe beam. Most important of all, the temperature measurements are independent of the ion acoustic wave amplitude, so that accurate measurements are possible without dealing with the complicated nature of the instability.

\section{c. Probe Beam Refraction}

It is important to estimate the refraction of the probe beam at the critical surface of the interaction laser (IR laser). The probe beam is incident onto the plasma with angel $\mathrm{u}_{0}$, where the plasma density increases $n$ the $x$ direction (the inset in Fig. 4). Using Snell's Law, the angle of incidence $u(x)$ of the probe beam at the IR critical surface is given by the following relation:

$$
\left(1-\frac{\omega^{2} p}{\omega^{2}}\right) \sin ^{2} \mathrm{u}_{\mathrm{u}}\left(\mathrm{x}_{\mathrm{c}}\right)=\sin ^{2} \mathrm{u}_{\mathrm{o}}
$$

where $\omega_{p}$ is the plasma frequency at the critical surface of the IR laser. The refraction of the probe beam $\left.\left(u\left(x_{c}\right)\right)-u_{0}\right) u_{O}$ is shown as a function of the incident angle in Fig. 2. For example, with a typical electron temperature of $T_{\mathrm{e}}=0.8 \mathrm{keV}$ and a $4 \omega$ probe beam, $u_{0}=$ $52^{\circ}$. Therefore, the refraction $\left(u\left(x_{c}\right)-u\right) / u$ is about $4.7 \%$, which produces an error $\Delta \mathrm{T}_{\mathrm{e}} / \mathrm{T}_{\mathrm{e}} \approx 6 \%$ in the electron temperature measurement. The effect of probe beam refraction is thus small for typical plasma parameters.

\section{d. Ion Acoustic Waves Excited by SBS}

It is expected that strong ion acoustic waves are excited by stimulated Brillouin scattering $(S B S)$. The wave number of the ion acoustic wave excited by SBS is small, $k B \simeq 2 \omega / c$. We obtain the ratio $\mathrm{kp}_{\mathrm{p}} / \mathrm{kB}=0.125 \mathrm{x} \mathrm{c} / \mathrm{ve}_{\mathrm{e}} \approx 3.2$ for $\mathrm{T}_{\mathrm{e}}=0.8 \mathrm{keV}$. This is equivalent to $k_{B} \lambda D_{\text {De }}(\lambda \mathrm{De}$ at the critical surface $)=0.08$. The dotted curve in Fig. $3(\mathrm{~B})$ corresponds to this case, which is clearly separated from the solid curve. The SBS ion wave may therefore disturb our measurement only when the third harmonic of the ion acoustic wave is strongly excited in a direction perpendicular to the IR laser propagation direction, 
which is improbable. Therefore, the ion acoustic waves excited by SBS will not disturb our measurements.

\section{e. Plasma Drift Velocity}

The ion acoustic dispersion relation is modified in a drifting plasma:

$$
\Omega_{\mathrm{i}} \simeq \mathrm{k}_{\mathrm{p}} \mathrm{C}_{\mathrm{s}}+\overrightarrow{\mathrm{k}}_{\mathrm{p}} \cdot \overrightarrow{\mathrm{U}}
$$

where $\vec{U}$ is the plasma drift velocity. The plasma is drifting mainly normal to the target surface. The ion waves are mainly driven along the electric field of the interaction laser, which is perpendicular to the drift direction. Thus, the drifting plasma should not strongly perturb our measurements. We will test the effect of the drift velocity by systematically varying the detection angle. 


\section{Experimental arrangements and the CTS experiments}

\section{Developments of the Multi-channel collenctive Thomson scattering system.}

We have constructed a five-channel detector system for the collective Thomson scattering. The system is especially designed to measure the lirge angle scattering from the ion acoustic decay instabilities. Figure 3 shows the schematics of the collective Thomson scattering system. The scattered signals are collected by the array of fivemirrors. We use optical fibers to guide the signal to a monochrometer. The diameters of the mirror and the optical fiber are $1 / 2$ inch and $100 \mu \mathrm{m}$. This detector measures the scattered signal from $200 \mu \mathrm{m}$ diameter. An interference filter is installed in each channel to protect the detector system from strong IR signal. The photographs of the improved version of the 5-channel detector system are shown in Fig. 4. Figure 4 (A), and 4 (B) show the top and side views. The improved version has capability of measuring the signal intensity using a diode array. The diode array is also be used for the calibration of the relative sensitivity of the each channel. The two-dimensional, angle and wavelength distribution is obtained with the film placed on the output of the monochrometer.

\section{Experiments}

Figure 5 shows a schematic of the experiment. The interaction IR laser is normally incident onto a planar target. The ion acoustic wave will be strongly excited by the IADI in the direction of the electric field of the IR laser. A probe beam is incident on the IADI region and is scattered by the ion acoustic wave. An array of small lenses (see Fig. 5) will be used to measure the angular distribution of the scattered signal.

We set the incident angle $\mathrm{u}_{0}=52^{\circ}$ of the probe beam as is shown in Fig. 5. The growth rate of the IADI will be proportional to $(\cos \theta)^{2}$, where $\theta$ is the angle between the wave vector of the ion acoustic wave and the electric field of the interaction laser. The ion wave vector will have an angular spread with a peak near $\theta=0^{\circ}$. The scattering angle is, about $115^{0}$ for $T_{e}$ is $0.6 \mathrm{keV}$, large angle scattering. We see from $\mathrm{Eq}$. (3) that $\mathrm{T}_{\mathrm{e}}$ is a well behaved function of the scattering angle for our typical experimental parameters. The signals are collected by an array of lenses with a solid angle $\Delta \Omega / 4 \pi=10^{-3}$ (one inch diameter lens placed at $20 \mathrm{~cm}$ from the target) and are fed to a monochromator. 
Simultaneous measurements for the scattering angle and the wave length of the scattered signal are obtained on film with two dimensional plots.

The alignment of the detector system, and the $4 \omega$ laser probe beam was made using 254 nm UV light from $\mathrm{Hg}$ lamp. We used a shadow method to accurately measure the alignment position. The UV signal of $254 \mathrm{~nm}$ was applied through the optical fiber. A tiny PM tube (Hamamatsu-R-1657) was placed just behind the $200 \mu \mathrm{m}$ diameter spherical target. The target position was slowly moved by a stepping motor. The signal amplitude was recorded on a synchronized chart recorder. Each mirror position was adjusted so that the dip of the signal was obtained when the target was at the center of the GDL chamber. The laser probe beam positioning was also made using this shadow method. The 5-channel collective Thomson scattering system was inštalled and calibrated in the GDL system. 


\section{Studies of the Ionic Charge State of High Z Plasma}

It is possible to estimate the ionic charge state $\mathrm{Z}$ near the critical surface by measuring the second harmonic emission. The ionic charge state $\mathrm{Z}$ is obtained by measuring the ion wave frequency $\Omega_{i}$ of the most unstable ion acoustic wave excited by the IADI, and using the following equation:

$$
\mathrm{Z}=\left(4.4 \times \frac{\Omega_{\mathrm{i}}^{2}}{\omega_{\mathrm{pe}}}\right)^{2} \times \frac{\mathrm{M}}{\mathrm{m}}-3 \frac{\mathrm{T}_{\mathrm{i}}}{\mathrm{T}_{\mathrm{e}}}
$$

The plasma density which corresponds to the $\mathrm{Z}$ measurements is obtained from the equation

$$
\frac{\mathrm{N}_{\mathrm{e}}}{\mathrm{N}_{\mathrm{c}}}=1-3(\mathrm{M} / \mathrm{m})\left(\Delta \lambda / \lambda_{2} \omega_{\mathrm{O}}\right)^{2} /\left(\mathrm{Z}+3 \mathrm{~T} \mathrm{i} / \mathrm{T}_{\mathrm{e}}\right)
$$

by measuring $\Delta \lambda$, and $\mathrm{Z}$ from the second harmonic emission due to IADI.

The measured plasma density which corresponds to the most unstable wave of the ion acoustic decay instability is about $n / n_{C}=0.86$.

A relatively weak intensity laser is incident on a planer target slightly above the threshold of the IADI. The second harmonic emissions are measured for $\mathrm{CH}, \mathrm{Mo}$, and gold targets with the irradiation of laser energy of $3 \times 10^{13} \mathrm{~W} / \mathrm{cm}^{2}$. The wavelength sift $\Delta \lambda$ (the difference between the $2 \omega_{0}$ peak and the Stokes peak) clearly decreases as the atomic number of the target increases, as shown in Fig. 6. The estimated value of the ionic charge state $\mathrm{Z}$ is shown in Fig.7 for $\mathrm{CH}$, Mo, and Gold targets.

We have also made LASNEX computer simulation calculations. The computer simulation results (with the flux number $\mathrm{f}=0.1$ ) agree very well with the experimental results. 


\section{The Ion Acoustic Decay Instability in High Power Laser Regime}

The ion acoustic decay instability with high power laser irradiation is quite different from the results with moderate intensity laser. The spectrum width of the Stokes peak increases with the laser intensity. Figure 8 shows a typical spectrum of the serond harmonic emission. The sharp peak at the left-hand side is the $2 \omega_{0}$ signal which is attributed to the emission from the electron plasma wave produced by the resonance absorption. The right hand side peaks are the Stokes modes by the IADI. We define Ip, and $I_{m}$ as the peak value of the Stokes mode, and the minimum value of the valley. Then the parameter $I_{\mathrm{p}} / \mathrm{I}_{\mathrm{m}}$ shows the degree of the spectrum broadening of the Stokes mode. If $I_{\mathrm{p}} / I_{\mathrm{m}}=1$, the specirum is flat, and if it is small, the s sectrum is sharp. The $\Delta \lambda_{\text {, distance }}$ between the $2 \omega_{0}$ and the Stokes peaks give the frejuency of the ion acoustic wave.

Figure 9 (A) show the $I_{p} / \mathbb{I}_{m}$ vs laser intensity. At laser power slightly above the threshold, the Stokes peak is sharp, so that the $I_{p} / I_{m}$ is small. The $I_{p} / I_{m}$ increases strongly with laser intensity. The $\mathrm{I} / \mathrm{I}_{\mathrm{m}}$ is nearly 1 when the laser intensity is larger than $1 \times 10^{14} \mathrm{~W} / \mathrm{cm}^{2}$. The plasma spectrum become very broad, indicating that turbulent like spectrum is observed. The figure 9 (B) shows $\Delta \lambda$ vs laser intensity. The $\Delta \lambda$ decreases with the laser intensity as is expected in turbulent like spectrum. 


\section{Studies of Laser Absorption lfor Mederate to High Power rrradiations}

\section{Hydrodynamic Equations}

Consider a planar plasma driven by a laser energy flux $(I(t))$ that is suddenly switched-on and then remains constant in time, $i$. e., $I(t)=0$, for $t<0$, and $I(t)=I_{0}$ for $t \geq 0$. We will develop self-similar solutions of the planar, one fluid hydrodynamic equations for each region. The equations for conservation of mass, momentum, and energy are

$$
\begin{aligned}
& \frac{\partial \rho}{\partial t}+\frac{\partial(\rho v)}{\partial x}=0 \\
& \frac{\partial v}{\partial t}+v \frac{\partial v}{\partial x}=-\frac{1}{\rho} \frac{\partial\left(\rho c^{2}\right)}{\partial x} \\
& \frac{3}{2} \rho \frac{\partial c^{2}}{\partial t}+\frac{3}{2} \rho v \frac{\partial c^{2}}{\partial x}+\rho c^{2} \frac{\partial v}{\partial x}=-\frac{\partial q}{\partial x}+\frac{\partial I}{\partial x}
\end{aligned}
$$

where $\rho$ is the mass density, $v$ is the fluid velocity, $c$ is the isothermal sound speed,

$c \equiv(P / \rho)^{1 / 2}, P$ is the pressure, $q$ is the heat flux, $I(x)$ is the spatially dependent laser energy flux, $I(x)=I_{0} \exp (-\tau(x)), I_{0}$ is the incident laser energy flux, and $\tau(x)$ is the inverse Bremsstrahlung optical depth of the plasma from $x^{\prime}=x$ to $x^{\prime}=\infty$, i.e.,

$$
\tau(x)=(x, \infty, \quad) \mathrm{Kib}_{\mathrm{ib}}\left(\mathrm{x}^{\prime}\right) \mathrm{dx^{ \prime }} .
$$

The inverse Bremsstrahlung absorption coefficient is given by

$$
\kappa_{\mathrm{ib}}(\mathrm{x}) \approx 3.4 \frac{\left(\rho / \rho_{\mathrm{c}}\right)^{2} \mathrm{Z} \ln \Lambda_{\mathrm{a}}}{\sqrt{1-\mathrm{n}_{\mathrm{e}} / \mathrm{n}_{\mathrm{c}}} \lambda_{\mu \mathrm{m}}^{2} \mathrm{~T}_{\mathrm{keV}}^{3 / 2}} \mathrm{~cm}^{-1}
$$

where $\rho_{c}$ and $n_{c}$ are the critical mass and number densities, ne is the electron number density, $\ln \Lambda_{a}$ is the Coulomb logarithm for inverse Bremsstrahlung absorption, $\lambda_{\mu m}$ is the laser light wavelength $(\mu \mathrm{m})$, and $\mathrm{T}_{\mathrm{keV}}$ is the electron temperature $(\mathrm{keV})$. The ponderomotive force is neglected since the radiation pressure due to the laser light is small compared to the plasma pressure for cases of interest. Radiation from the plasma and the inertial force due to ablative acceleration of the target have also been neglected. These terms are generally small for parameters of interest to laser pellet fusion, especially for shorter wavelength lasers. The electron and iun temperatures are assumed to be equal, so that $c^{2}=(Z+1) T /\left(A M_{p}\right), Z$ is the ionic charge state, $A$ is the atomic weight, and 
$M_{p}$ is the proton mass. The heat flux is given by the smaller of the classical heat flux and a phenomenological model for limited heat flux, i.e.,

$$
q=\operatorname{Min}\left[K_{0} T_{e}^{5 / 2}\left|\frac{\partial T_{e}}{\partial x}\right|, f n_{e} T_{e}\left(T_{e} / m\right) 1 / 2\right] \frac{\frac{\partial T_{e}}{\partial x}}{\left|\frac{\partial T_{e}}{\partial x}\right|}
$$

where the heat flux coefficient is $\mathrm{K}_{0}=1.8 \times 10^{29} / \mathrm{Z} \ln \Lambda, \mathrm{cm}^{-1} \mathrm{sec}-1 \mathrm{keV}-5 / 2, \ln$ $\Lambda$ is the Coulomb logarithm, $m$ is the electron mass, and $f$ is the flux limiter.

\section{Conduction Region Model}

We will show a-posteriori that the laser energy is deposited predominantly in the corona for moderate to high laser powers and typical pulse widths. We will therefore ignore laser deposition and develop a self-similar solution for the conduction region. We take the similarity variable to be, $s=\left(x-x_{a}\right) / \Delta_{S}(t)$, where, $\Delta_{S}(t)$ is the width of the conduction region, $\Delta_{\mathrm{S}}=\mathrm{x}_{\mathbf{S}}-\mathrm{x}_{\mathrm{a}}$, to be determined. Guided by the LASNEX simulations, we assume that that the dependent variables can be written: $\rho(x, t)=\rho_{S}(t) R(s), v(x, t)=$ $c_{s}(t) V(s)$, and $c^{2}(x, t)=c_{s}^{2} E(s)$. The boundary conditions are: $E(0)=V(0)=1 / R(0)=0$, and $E(1)=V(1)=R(1)=1$. The hydrodynamic equations become

$$
\begin{gathered}
r R-s(n+1) \frac{d R}{d s}=-\frac{c_{s} t}{\Delta_{s}} \frac{d(R V)}{d s} \\
(r+n) R V-s(n+1) \frac{d(R V)}{d s}=-\frac{c_{s} t}{\Delta_{s}} \frac{d(R V 2+R E)}{d s} \\
\frac{3}{2}\left[2 n E-s(n+1) \frac{d E}{d s}\right]+\frac{3}{2} \frac{c_{S} t}{\Delta_{s}} V \frac{d E}{d s}+\frac{c_{S} t}{\Delta_{s}} E \frac{d V}{d x}=\frac{c_{s} t}{\Delta_{s}} \frac{K_{0} T_{s}^{7 / 2}}{\rho_{s} c_{s}^{3} \Delta_{s}} \frac{1}{R} \frac{d\left[E^{5 / 2} \frac{d E}{d s}\right]}{d s}
\end{gathered}
$$

where

$$
\frac{\partial \rho_{s}}{\partial t}=\frac{r \rho_{s}}{t}
$$

and

$$
\frac{\partial c_{s}}{\partial t}=\frac{n c_{s}}{t} .
$$

Eqs. (21) - (23) are self-similar ( i. e., not explicitly a function of time and space) if: The factor, $\left(c_{S} t\right) / \Delta_{S}$ and the factor, $K_{0} T_{S}{ }^{7 / 2} /\left(\rho_{S} \alpha_{S}^{3} \Delta_{S}\right)$ are not a function of time. Using 
Eqs. (24) and (25) the second condition results in, $r=3 n-1$. Another condition results from requiring that the absorbed laser energy is the plasma energy source, i.e.,

$$
\mathrm{I}_{\mathrm{O}}=\beta p_{\mathrm{s}} \mathrm{c}_{\mathrm{s}}^{3}
$$

where $\beta$ is a constant that depends on the plasma spatial profile. This results in, $r=-3 n$, or we find, $r=-1 / 2$ and $n=1 / 6$.

The solution of Eqs. (2i) - (23) require the solution of a set of four first order, nonlinear differential equations that are very difficult to solve. However, guided by the LASNEX simulations we find the following approximate solutions: $\mathrm{E} \approx \mathrm{s} 2 / 5, \mathrm{~V} \approx \mathrm{s} 3 / 5$, and $R \approx 1 / \mathrm{V}$. The approximate solutions for the conduction region are therefore

$$
\begin{aligned}
& T \approx T_{s}\left[\left(x-x_{a}\right) / \Delta_{s}\right]^{2 / 5} \\
& v \approx c_{s}\left[\left(x-x_{a}\right) / \Delta_{s}\right]^{3 / 5} \\
& \rho \approx 1 / v
\end{aligned}
$$

Since the local sound speed (c) is proportional to $T^{1 / 2}$, we see that one important prediction is that the Mach number $(M=v / c)$ is equal to the normalized temperature. The predicted normalized temperature ( from Eq. (27)) is in agreement with temperature (circles) and Mach number (diamonds) from LASNEX simulations . (The width of the conduction region was set equal to the LASNEX result $(0.026 \mathrm{~cm})$ for this figure.) The agreement is good for the three laser wavelengths shown $(\lambda=0.35,0.53$, and $1.059 \mu \mathrm{m})$. We also see that the width of the conduction region is independent of wavelength. This agrees with results from the self-similar theory as will be shown below. In the LASNEX simulations, a thick aluminum slab was irradiated with a peak incident laser energy flux, $I_{0}=1 \times 10^{15} \mathrm{~W} / \mathrm{cm}^{2}$. The results are shown at the peak of the Gaussian pulse (pulse width, $t_{L}=2$ ns ( Full Width Half Maximum, FWHM )). The flux limit was, $f=0.1$, for these calculations, and the heat flux for the conduction region was low enough so that the heat flux was below the flux limit.

\section{Corona region Model}

The well known similarity solution for the expansion of an isothermal plasma into vacuum is used for the corona region, i.e.

$$
\begin{aligned}
& \rho(x, t)=\rho_{S} \exp \left(-\left(x-x_{S}\right) / c_{S} t\right) \\
& v(x, t)=c_{S}+\left(x-x_{S}\right) / t
\end{aligned}
$$

The range of validity of this assumption is determined a-posteriori.. The isothermal rarefaction is maintained by a mass flux $\left(\rho_{S} c_{S}\right)$ from the subsonic region and an energy flux $\left(\rho_{\mathrm{S}} \mathrm{c}_{\mathrm{S}}^{3}\right)$ due to laser absorption.

A. Heat Flux in the Corona 
The heat flux in the corona can be found by integrating Eq. (17) $\left(\int_{x}^{\infty}() d x^{\prime}\right)$ to obtain

$$
q(x)=-\alpha(x) I_{0}+\rho(x) c(x)^{3}
$$

where $\alpha(x)$ is the laser absorption in the plasma from $x$ to $\infty$, i.e.,

$\alpha(x)=1-\exp (-\tau(x))$. The terms with the derivative of the temperature (first and second terms in Eq. (17)) were neglected compared to the third term. It will be shown aposteriori that these terms are sr. 11 in the corona. We showed that in the steady state conduction region model the tempe ature must be a maximum at the sonic surface. Thus, the heat flux is zero at the sonic surface and in the isothermal corona. In our present time dependent model the heat flux is not zero in the corona and the heat flux at the sonic surface is (Eq. (31) evaluated at $\mathrm{x}=\mathrm{x}_{\mathbf{S}}$ )

$$
q_{s}=-\alpha_{s} I_{0}+\rho_{s} c_{s}^{3}
$$

Eq. (32) can be reduced to the steady state model by setting $q_{s}=0$, so that $\alpha_{S}=\rho_{s} c_{S}^{3} / I_{O}$, which along with $I_{O}=4 \rho_{s} c_{\xi}^{3}$, from the steady state model gives $\alpha_{S}=1 / 4$ in agreement with the steady state model.

\section{Width of the Conduction region}

The key features of our self-similar model are that the conduction region is subsonic and evolves in time. One estimate for the width of the subsonic region of a laser heated plasma, $\Delta_{\mathbf{S}}$, is the width of the subsonic region in the expansion of an isothermal plasma, $\Delta_{\mathrm{is}}=\mathrm{c}_{\mathrm{S}} \mathrm{t}$. The subsonic region in a laser heated plasma must be smaller than this because the subsonic region in a laser heated plasma is certainly not isothermal. In our model, the sonic surface is the boundary between the conduction and corona regions. The width of the conduction region is found by requiring that the heat flux in the coron: (Eq. (32)) and the heat flux in the conduction region are equal at the sonic surface. Eq. (27) for the temperature in the conduction region is used to find the heat flux at the sonic surface, $\mathrm{q}_{\mathrm{S}}=-(2 / 5) \mathrm{K}_{\mathrm{O}} \mathrm{T}_{\mathrm{S}}{ }^{7 / 2} / \Delta_{\mathrm{s}}$. Substituting this equation into Eq. (32 and solving for $\Delta_{\mathrm{S}}$ results in

$$
\Delta_{S}=\frac{2}{5} \frac{K_{0} T_{S} 7 / 2}{\alpha_{S} I_{0}-\rho_{S}{ }^{3}}
$$

Another equation for the width is found by matching the spatial derivative of the velocity at the sonic surface. From the approximate solution for the subsonic region( Eq. (28)), the derivative of the velocity is

$$
\frac{\partial v}{\partial x}\left(x \leq x_{S}\right) \approx 0.6 \frac{c_{s}}{\Delta_{S}},
$$


and, $\frac{\partial v}{\partial x}\left(x \geq x_{S}\right) \approx \frac{1}{t}$ from the supersonic region (Eq. (30)). Another equation for the width of the subsonic region is therefore

$$
\Delta_{\mathrm{S}} \approx 0.6 \mathrm{c}_{\mathrm{S}} \mathrm{t}
$$

The sonic surface moves outward relative to the ablation surface at a velocity of $\approx 0.6 \mathrm{c}_{S}$.

\section{Plasma Properties}

One condition on the plasma properties at the sonic surface is obtained by equating the absorbed laser flux, $\mathrm{I}_{\mathrm{o}} \mathrm{t}$, to the total plasma energy/area ${ }$ The total plasma energy/area for the conduction region is obtained by using Eqs. (27) - (29) to form the energy density and then integrating over the conduction region. This energy/area is added to the energy/area for the corona $\left(4 \rho_{s} c_{\xi}^{3}\right)$, resulting in

$$
\mathrm{I}_{0} \approx 5.5 \rho_{\mathrm{s}} \mathrm{c}_{\mathrm{s}}^{3}
$$

Another equation is obtained by equating Eq. (33) to Eq. (34), resulting in

$$
\mathrm{c}_{\mathrm{S}} \mathrm{t} \approx 0.8 \frac{\mathrm{K}_{\mathrm{O}} \mathrm{T}_{\mathrm{S}} 7 / 2}{\mathrm{I}_{\mathrm{O}}}
$$

In the spirit of our model, we assumed that the laser energy was deposited in the corona ( $\alpha_{S} \approx 1$ ). The resulting density, temperature and mass ablation rate at the sonic surface are

$$
\begin{aligned}
& \rho_{\mathrm{S}}=1.7 \times 10^{-9}\left[\frac{\mathrm{I}_{0}}{\mathrm{Zt} \ln \Lambda}\right]^{1 / 2}\left[\frac{\mathrm{A}}{\mathrm{Z}+1}\right]^{7 / 4} \mathrm{gm} / \mathrm{cm}^{3} \text {, } \\
& \left.\mathrm{T}_{\mathrm{S}}=1.1 \times 10^{-5}\left[\mathrm{Z} \mathrm{I}_{\mathrm{O}} \mathrm{t} \ln \Lambda\right]\right]^{1 / 3}\left[\frac{\mathrm{Z}+1}{\mathrm{~A}}\right]^{1 / 6} \mathrm{keV} \\
& \rho_{\mathrm{S}} \mathrm{c}_{\mathrm{S}}=1.7 \times 10^{-4} \frac{\mathrm{I}_{\mathrm{O}}^{2 / 3}}{[\mathrm{Zt} \ln \Lambda]^{1 / 3}}\left[\frac{\mathrm{A}}{\mathrm{Z}+1}\right]^{7 / 6} \frac{\mathrm{gm}}{\mathrm{cm}^{2} \mathrm{sec}} \text {, }
\end{aligned}
$$

Here, the units are: $I_{0}\left(W / \mathrm{cm}^{2}\right), \lambda(\mu \mathrm{m})$, and $t(\mathrm{~ns})$. The ablation pressure is

$$
\mathrm{P}_{\mathrm{a}}=2 \rho_{\mathrm{s}} \mathrm{c}_{\mathrm{s}}^{2}=3.4 \times 10-11 \frac{\mathrm{I}_{0}^{5 / 6}}{(\mathrm{Z} \ln \Lambda \mathrm{t})^{1 / 6}} \frac{\mathrm{A}^{7 / 12}}{(\mathrm{Z}+1)^{7 / 12}} \mathrm{Mbar} .
$$

The key feature of these solutions is that the plasma properties at the sonic surface and the ablation pressure are not a function of laser wavelength. The mass density (Eq. (37)), temperature (Eq. (37) ) at the sonic surface, and the ablation pressure (Eq. (40)) are compared to LASNEX simulations (circles, $\lambda=1.059 \mu \mathrm{m}$, crosses, $\lambda=0.53 \mu \mathrm{m}$, 
triangles, $\lambda=0.35 \mu \mathrm{m}$ ) at the peak of the laser pulse. The target is a thick aluminum slab (in the model, the aluminum was taken as fully ionized in agreement with the LASNEX results). The width of the Gaussian laser pulse is, $t L=2 \mathrm{~ns}$ (FWHM). The laser pulse width was reduced by a factor of 0.53 to compare our constant laser energy flux model to the Gaussian laser pulse used in the LASNEX simulations. (The factor is chosen so that the absorbed laser energy used in the model calculations is the same as in the LASNEX simulations.) Calculations were made for peak laser energy fluxes of, $I_{O}=1 \times 10^{14}$ and 1 $x 10^{15} \mathrm{~W} / \mathrm{cm}^{2}$ and laser wavelengths, $\lambda=0.35,0.53$, and $1.059 \mu \mathrm{m}$. The LASNEX results are in gond agreement with the self-similar theory. The steady state theory gives results that disagree by a factor of 2 to 3 .

The time dependence from the self-similar theory agrees with the L.ASNEX simulations . The laser pulse in the LASNEX simulations was Gaussian ( FWHM = 2ns ) until the peak, then constant in time.

In agreement with the theory, LASNEX calculations of the spatially dependent pressure are to be closely independent of wavelength $(\lambda=0.35 \mu \mathrm{m}$ and $0.53 \mu \mathrm{m}$ are shown ) over the whole plasma. The pressure agreed in the conduction region and was about $20 \%$ lower in the corona. This reduced pressure is a direct result of :imiting the heat flux as can be seen by solving Eq. (31) for the pressure, $P=\left(q+\alpha, I_{0}\right) / c$. 


\section{References}

1. K. Mizuno, W. Seka, R. Bahr, R.P Drake, P.E. Young, J.S. De Groot, and K. Estabrook, "Ion Acoustic Parametric Decay Instabilities in Laser Plasma Interactions," Laser Interaction and Related Plasma Phenomena, vol. 9, edited by Hora and G.H. Hiley.

2. K. Mizuno, P.E. Young, W. Seka, R. Bahr, J.S. De Groot, R.P. Drake, and K.G. Estabrook, Phys. Rev. Lett. 65, 428 (1990).

3. C. Yamanaka et al, Phys. Rev. Lett 30, 594 (1973).

4. J.L. Bobin, et al., Phys. Rev. Lett., 594 (1973)

5. K. Eidman and R Sigel, Phys. Rev. Lett. 34, 799 (1975)

6. N.G. Basov, et al., Sov. Phys. JETP 49, 1059 (1979)

7. X. Zhihan, et al., J. Appl. Phys. 54, 4902 (1983)

8. Y. Takada, et al., Phys. Fluids 31, 692 (1988)

9. P.D. Carter, S.M. Sim, and T.P Hughes, Opt. Commun, 27, 423 (1987)

10. K. Tanaka, et al., Phys. fluids 27, 2187 (1984)

11. K. Mizuno, J.S. De Groot, and K.G. Estabrook, Phys. Rev. Lett. 52, 271 (1984); Phys. Fluids 29, 568 (1968)

12. K. Mizuno, J.S. De Groot, W. Woo, P.W. Rambo, and K.G. Estabrook, Phys. Rev. 38, 4333 (1988).

13. K. Mizuno, F. Kehl, and J.S. De Groot, Phys. Rev. Lett. 56, 2184 (1986)

14. K. Mizuno and J.S. De Groot, The Proceedings of the 1987 International Conference on Plasma Physics, Kiev, USSR, 654, (1987) (edited by A.G. Stenko)

15. K. Mizuno and J.S. De Groot, "Anomalous Thermal Electron Heating and Heat Transport Inhibition Due to Parametric Instability," Phys. Fluids (in press)

16. J.S. De Groot, et al., The Proceedings of the Seventh International Workshop on Laser Interactions and Related Plasma Phenomena, Plenum Press (1987)

17. P.W. Rambo, W. Woo, J.S. De Groot, and K. Mizuno, Phys. Fluids 27, 2234 (1984)

18. K. Estabrook and W.L. Kruer, Phys. Fluids 26, 1888 (1983)

19. J.E. Bernard and J. Meyer, Phys. Fluids 29, 2313 (1986)

20. H.A. Baldis, D.M. Villeneuve, and C.J. Walsh, Can. J. Phys. 64, 961 (1986) 


\section{Figure Captions}

Fig. 1 Theoretical results of $\mathrm{T}_{\mathrm{e}} \mathrm{vs}$ the scattering angle.

Fig. 2 The refraction of the probe laser beam.

Fig. 3 The schematics of the collective Thomson scattering device.

Fig. 4 The pictures of the 5-channel detector . (A) top view, and (B) side view.

Fig. 5 The schematics of the collective Thomson scattering experiments.

Fig. 6 The spectrum of the $2 \omega$ emission for $\mathrm{CH}, \mathrm{Mo}$, and Au targets. The inst shows the normalized ion acoustic wave frequency vs atomic number.

Fig. 7 The estimated values of the ionic charge state vs atomic number.

Fig. 8 A typical second harmonic spectrum, which is shown define the parameters of $I_{p}$, and $I_{m}$.

Fig. 9 (A) The measurement of the spectrum broadening. The $I_{m} / I_{p}$ vs laser intensity. $\mathrm{CH}$ target.

(B) The measurements of the frequency shift. The $\Delta \lambda$ vs laser intensity. 


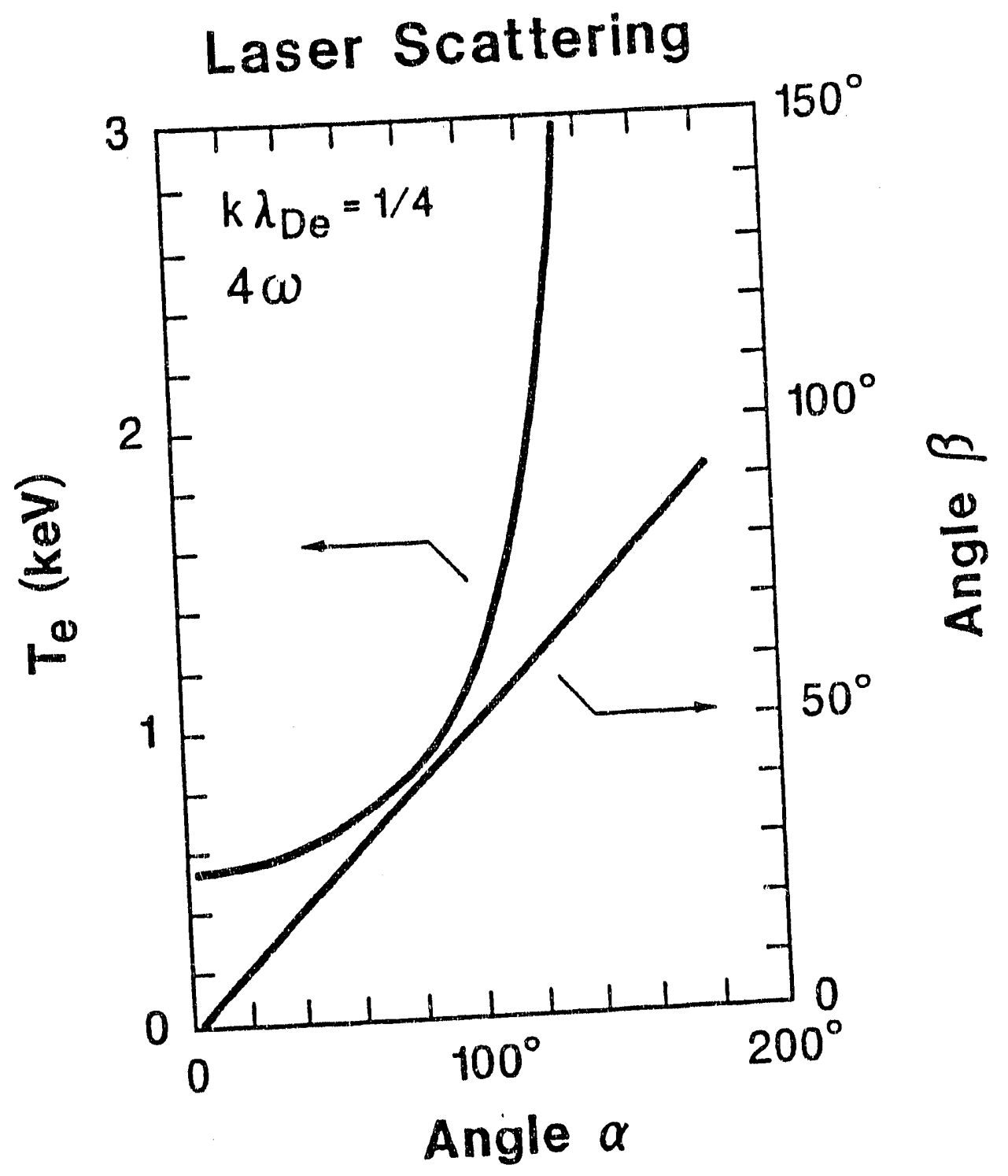

Fig. 1 


\section{REFRACTION}

Angle of probe beam at the critical surface of IR laser

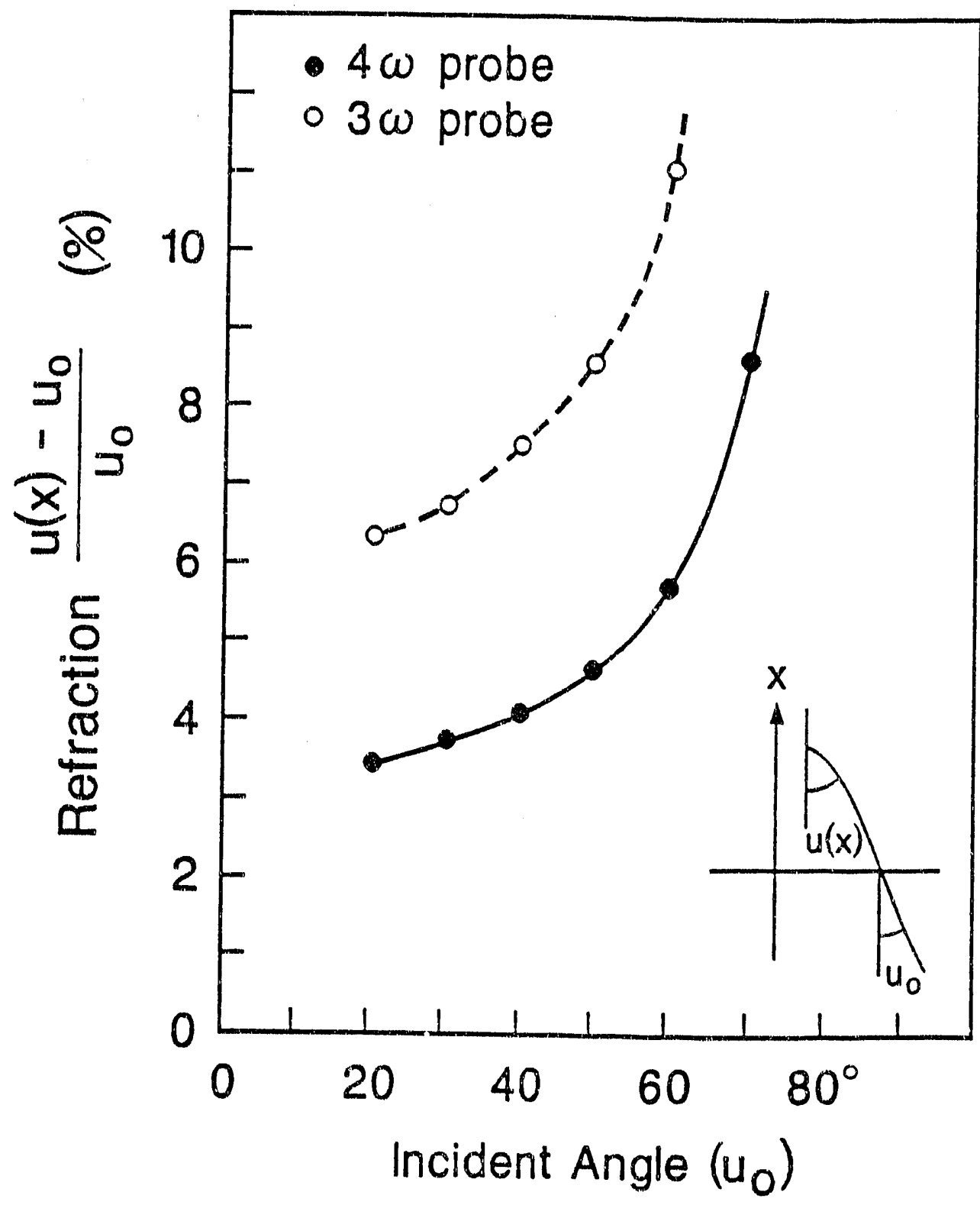

Fig. 2 


\section{Five-Channel Detector System for the Collective Thomson Scattering}
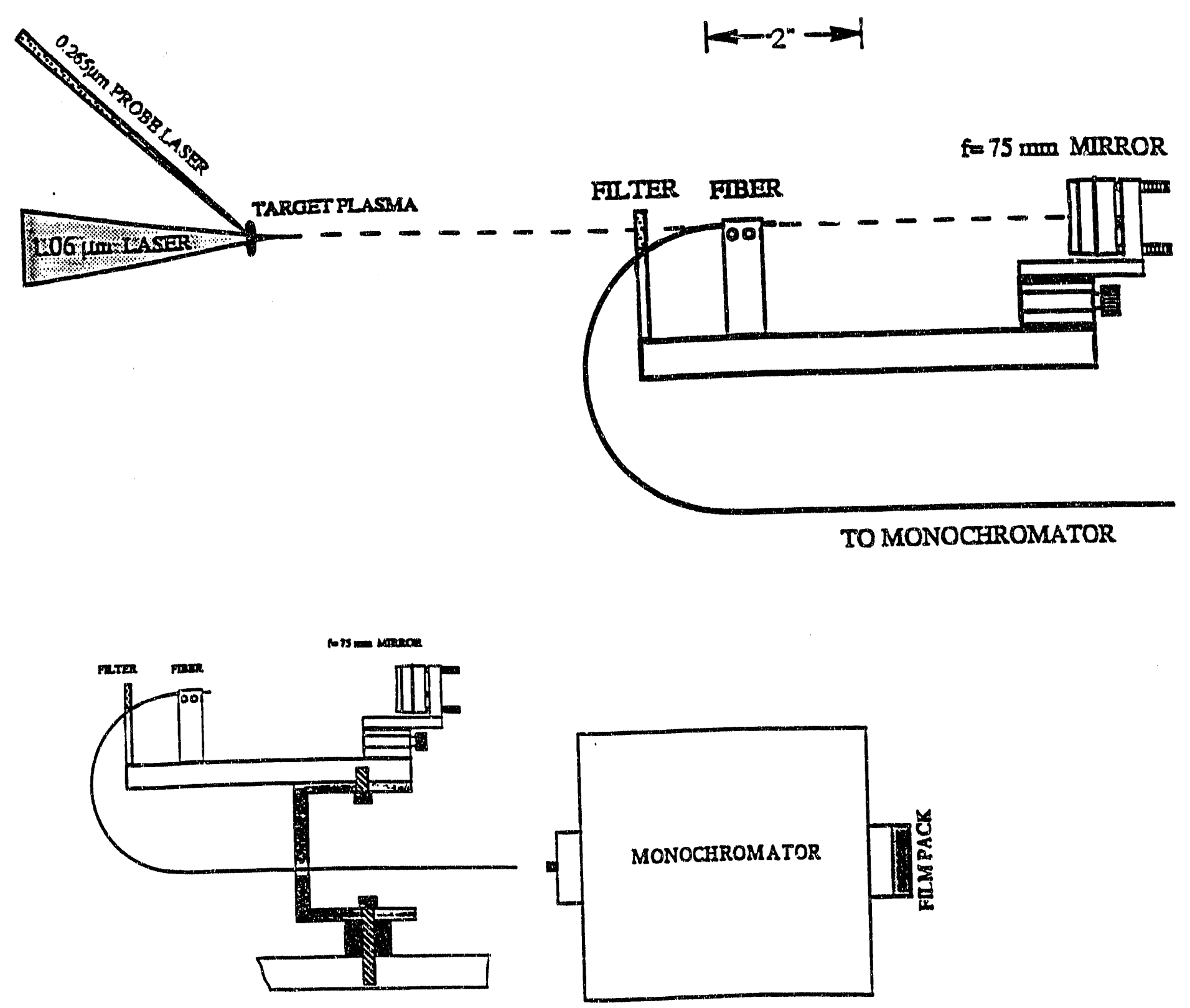

Fig. 3 
(A)

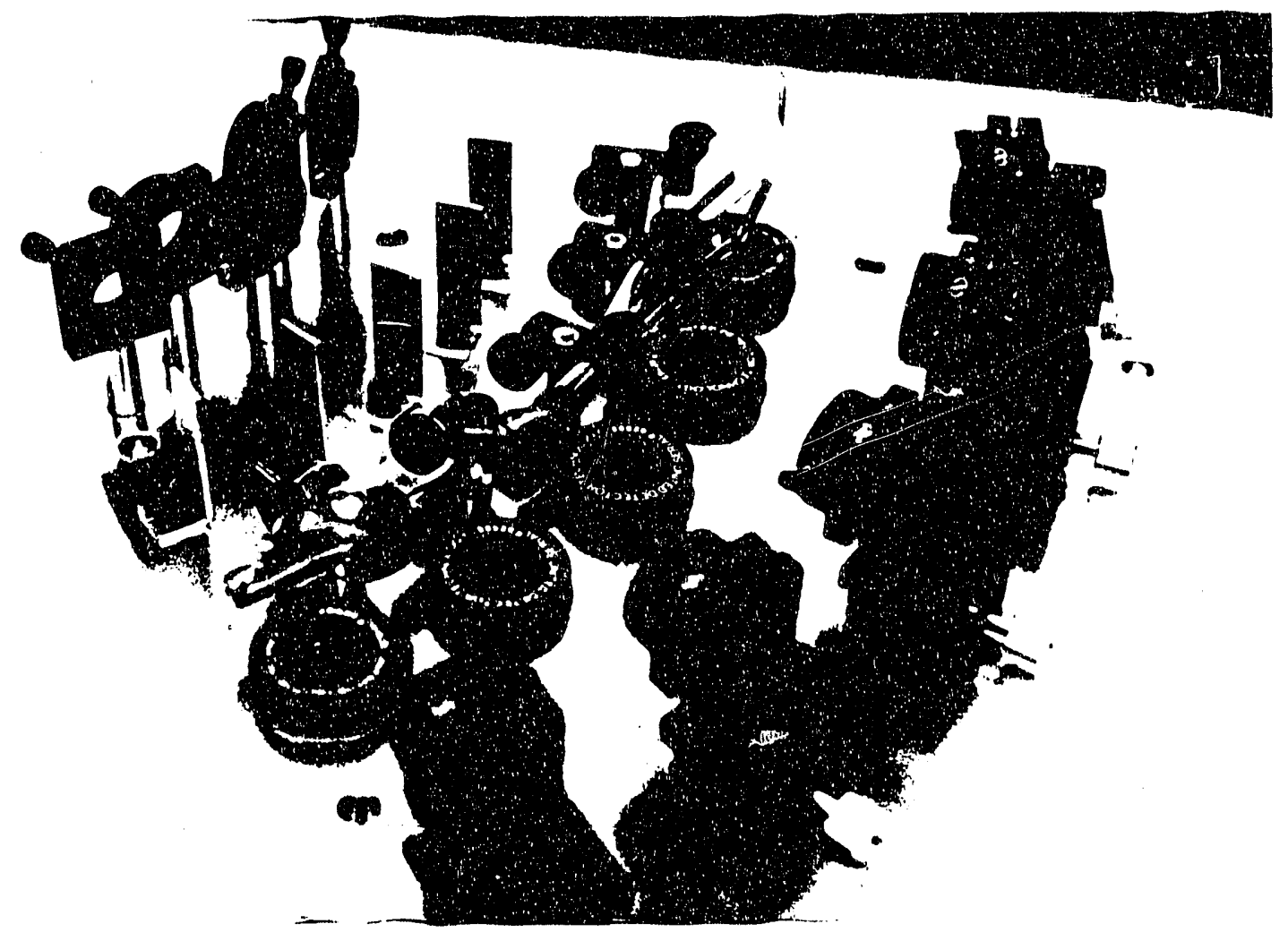

(B)

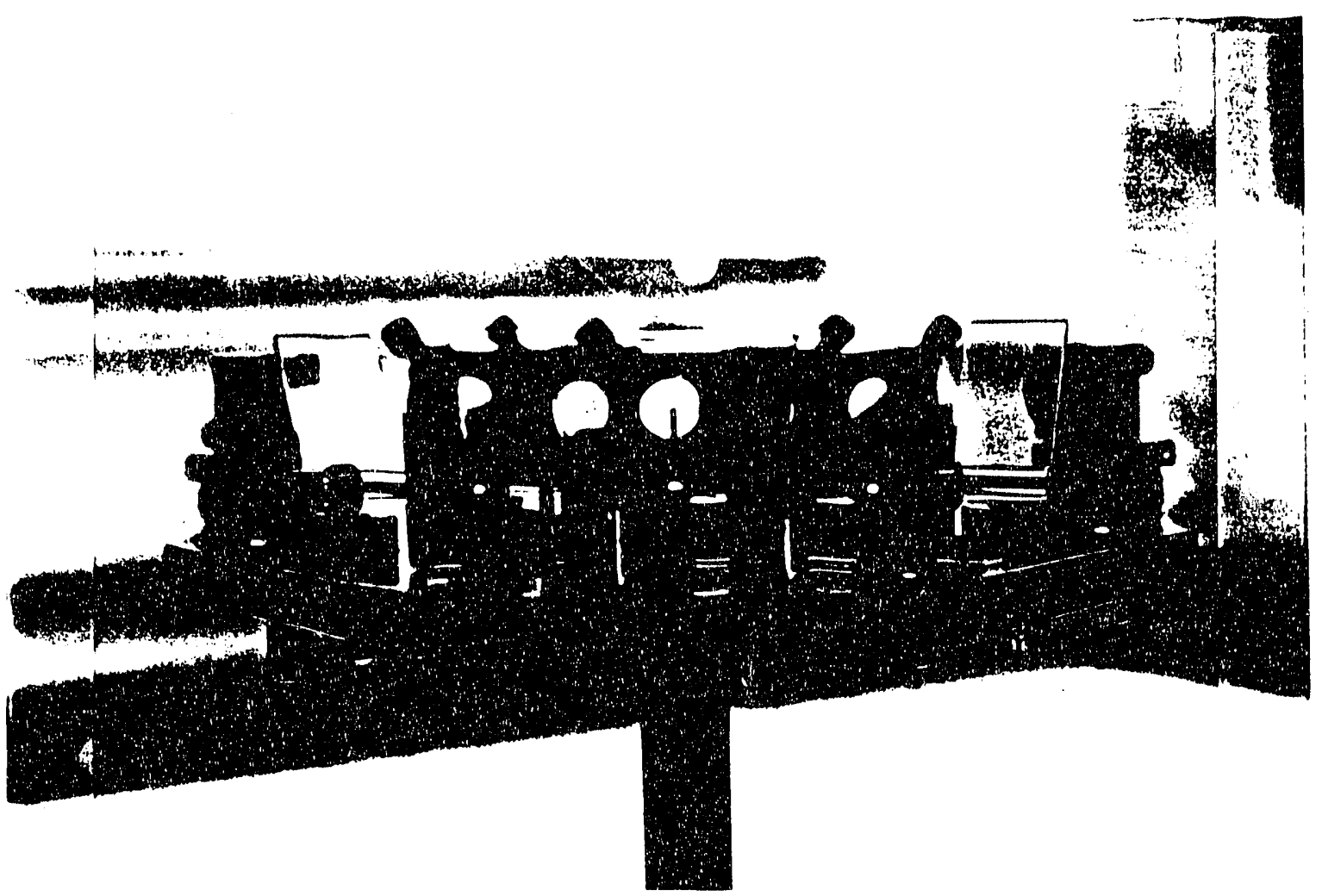

Fig. 4 
The Schematics of the Collective Thomson Scattering

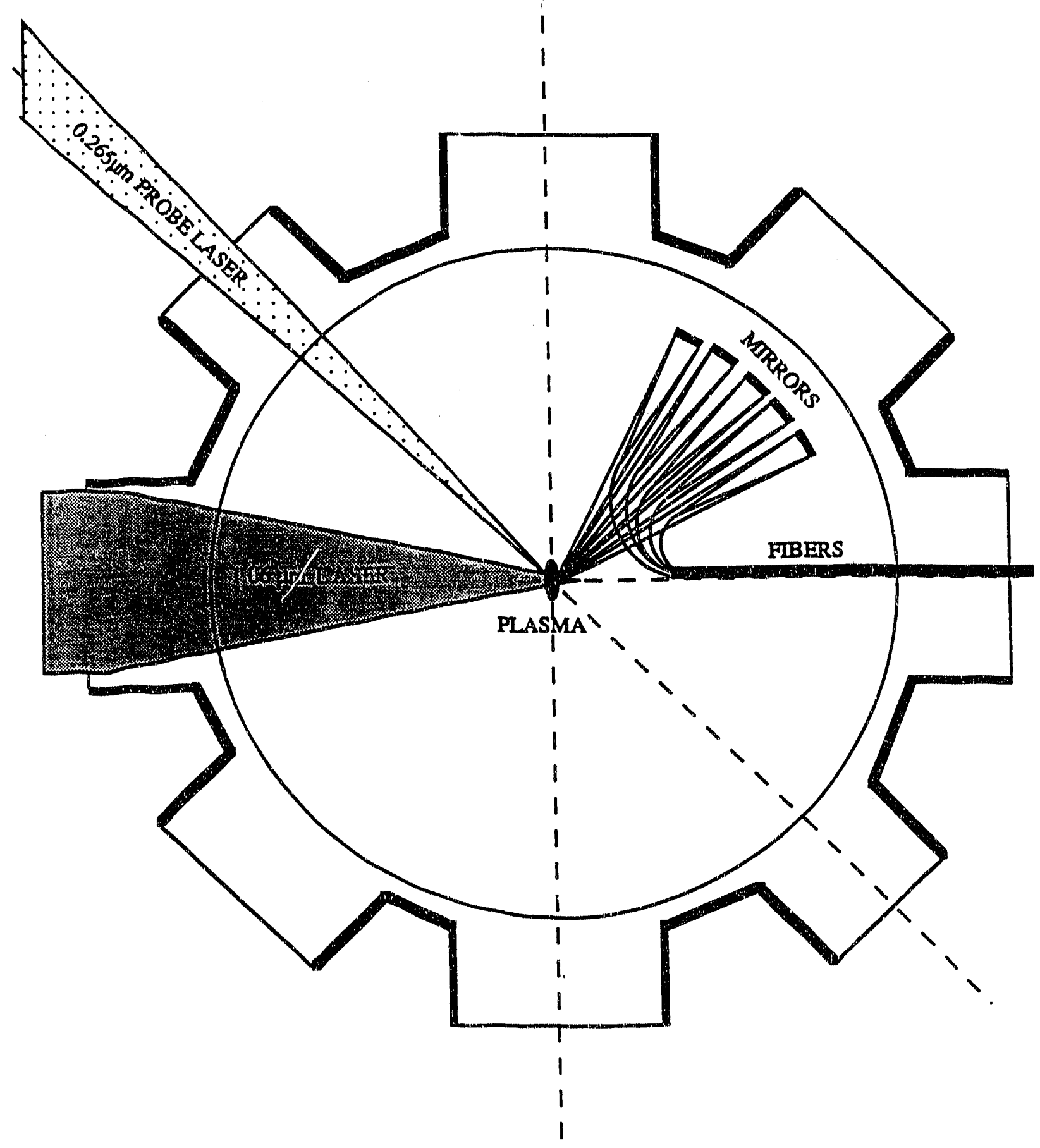

Fig. 5 


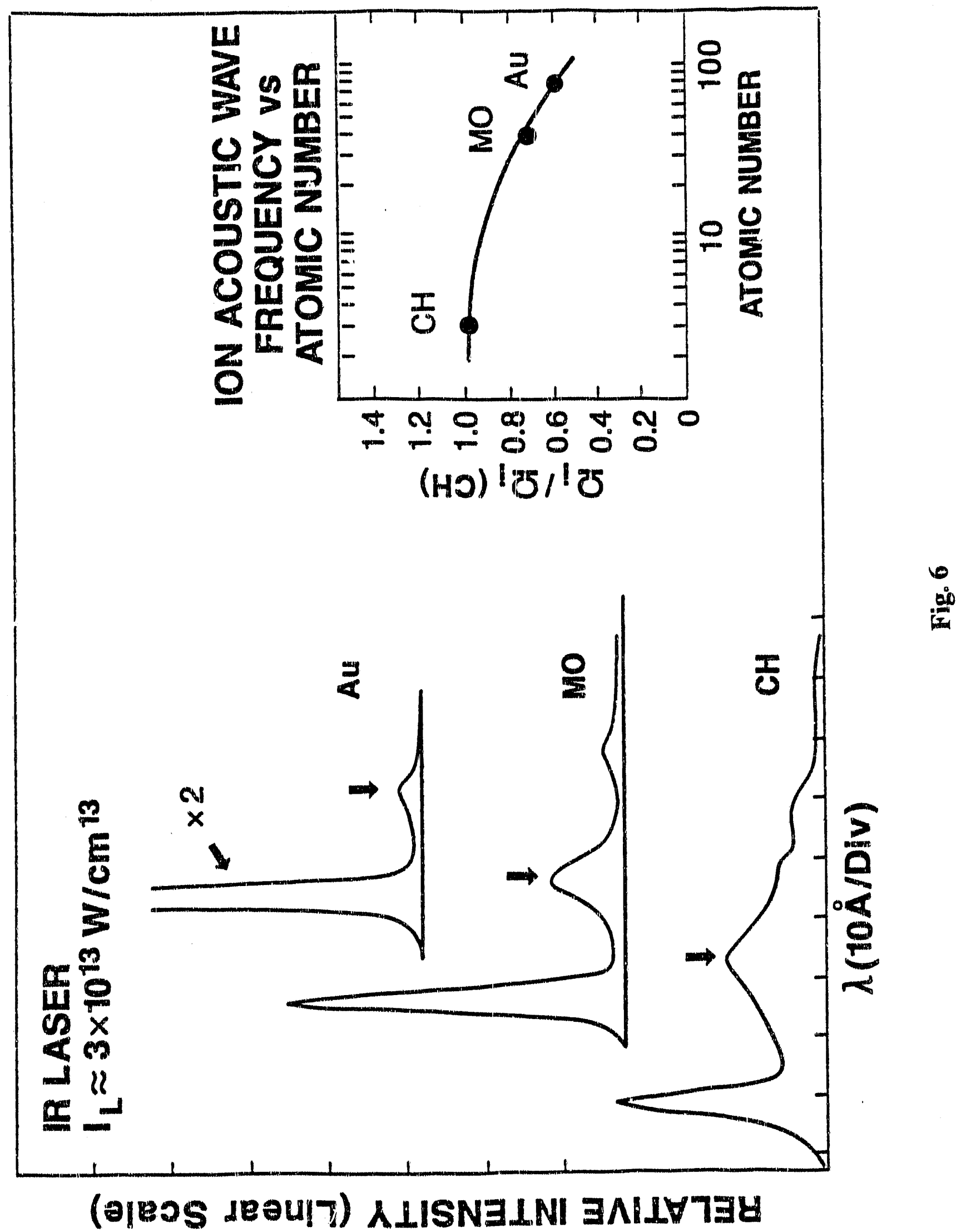




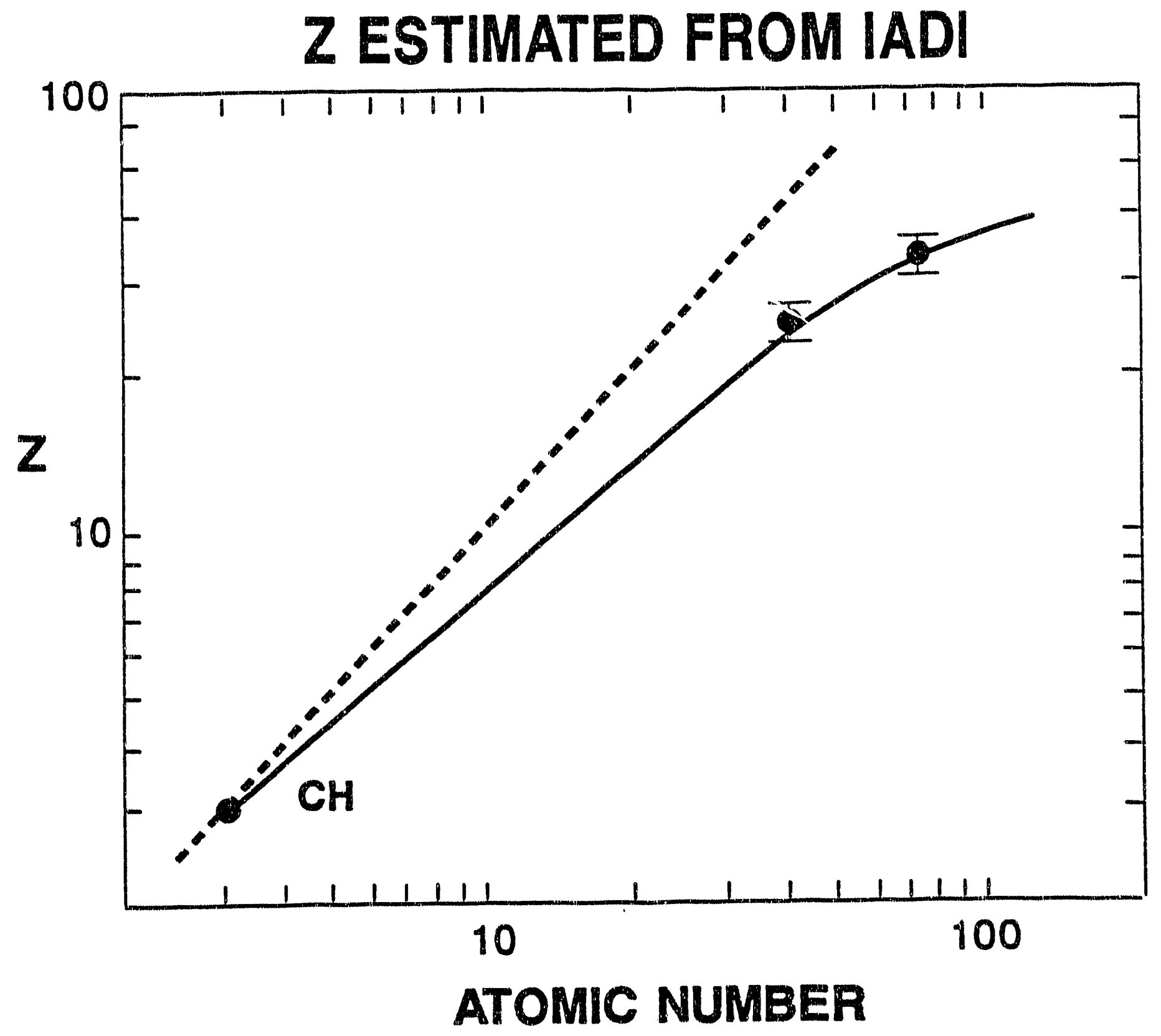

Fig. 7 


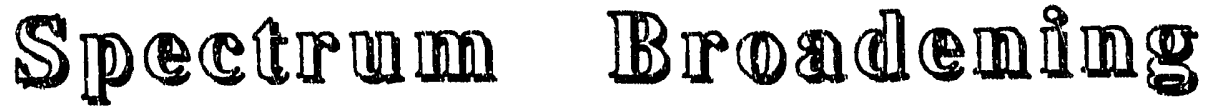

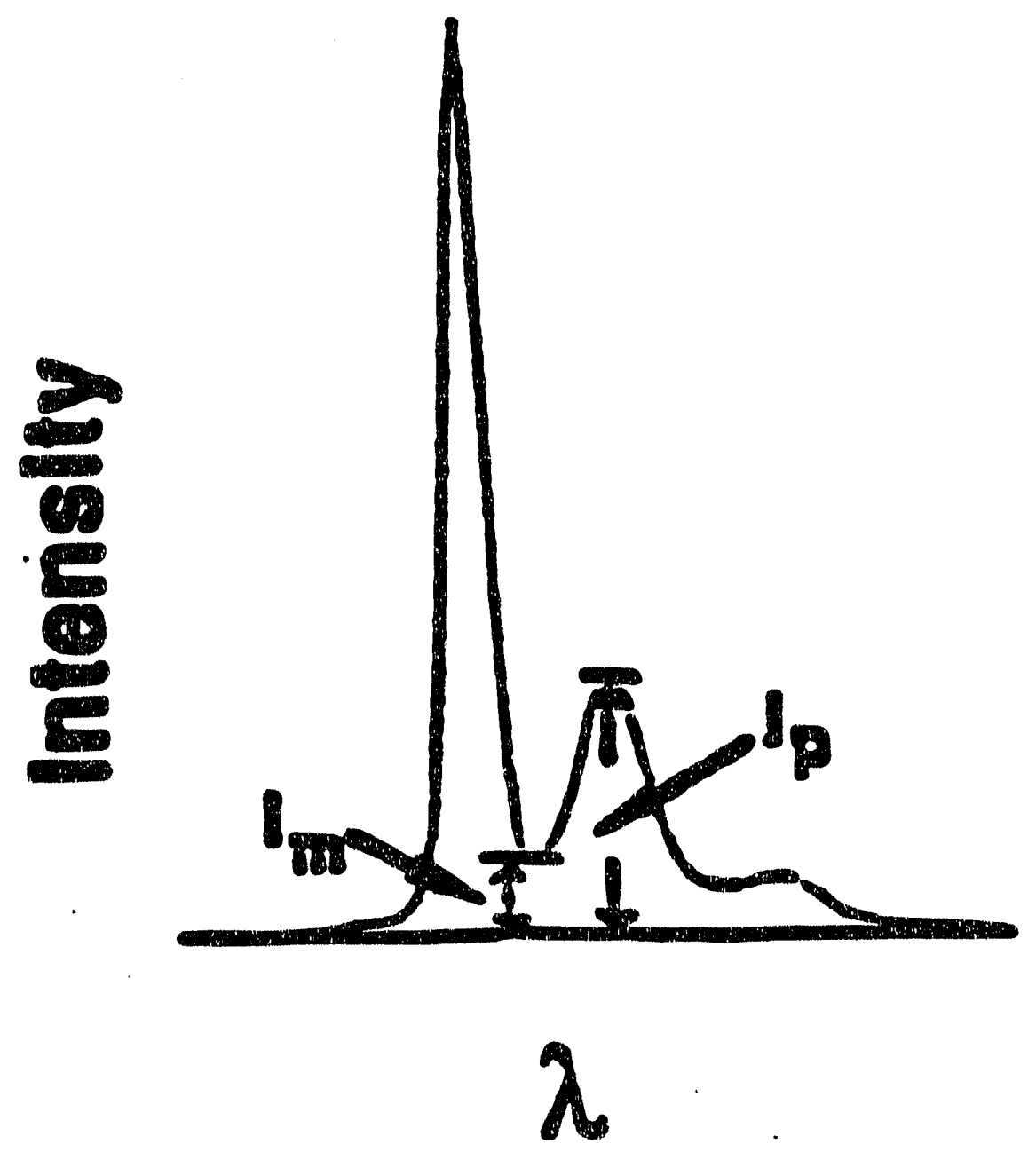

Fig. 8 

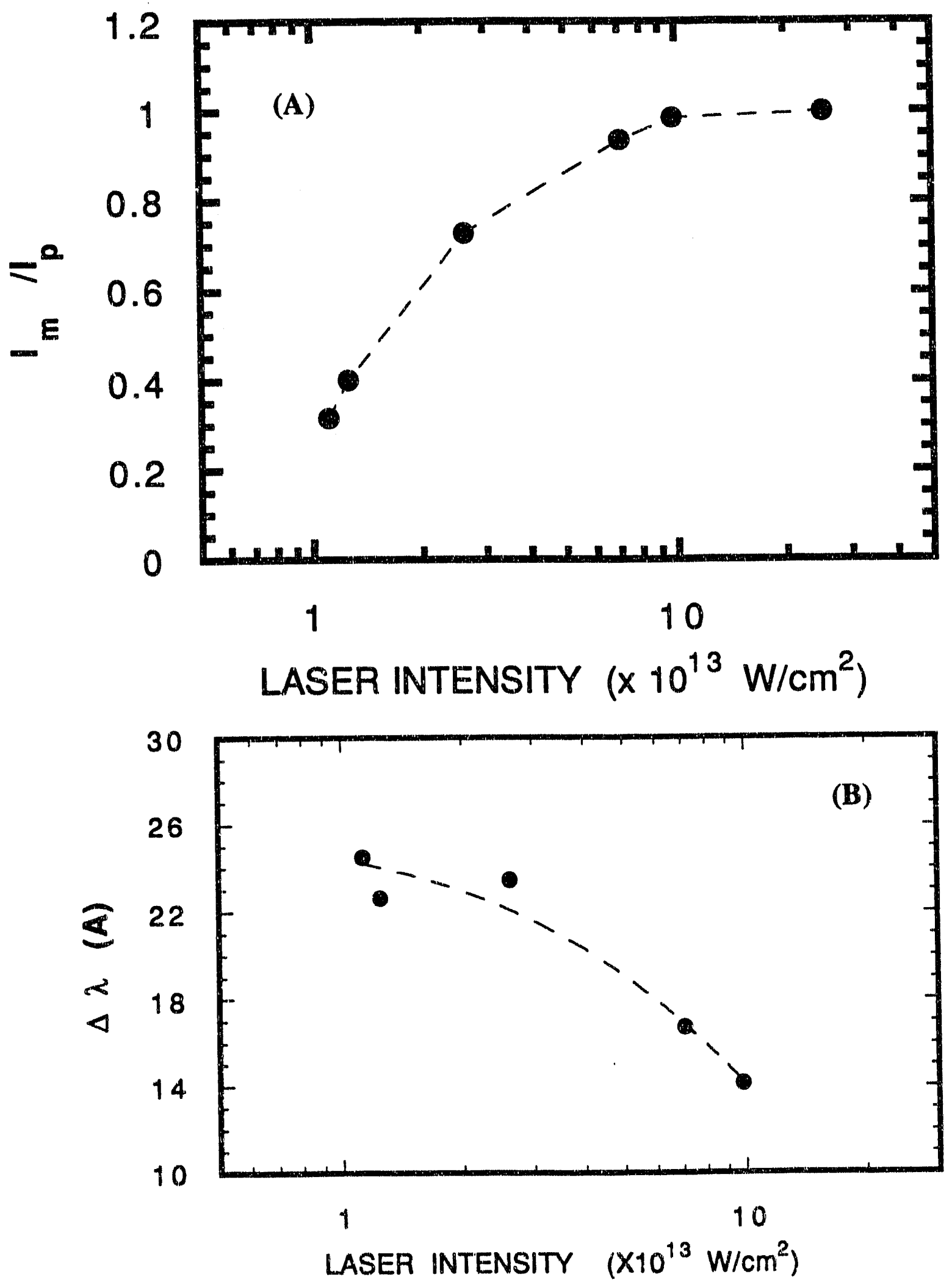

Fig. 9 


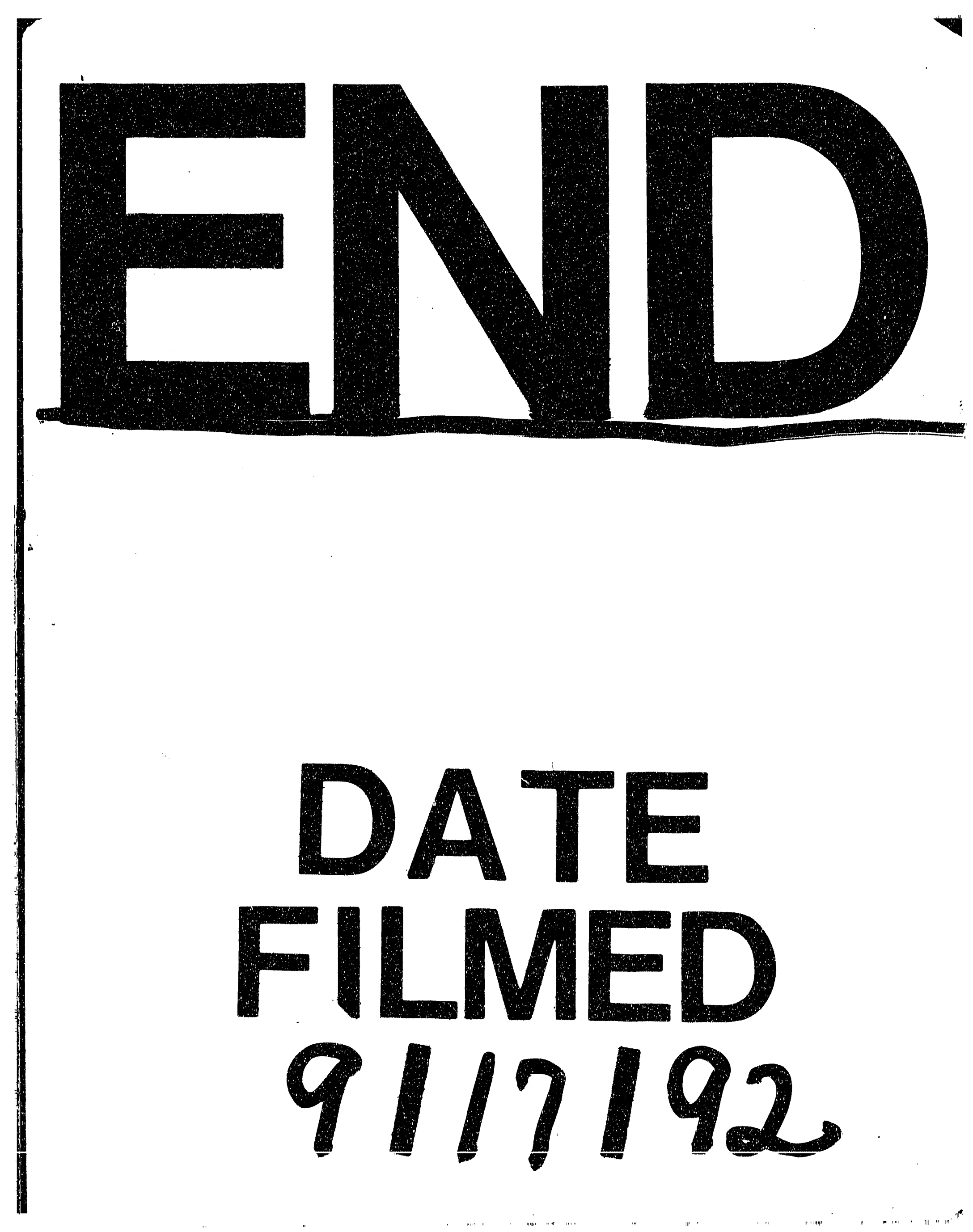


